\title{
On the Thermodynamic Equilibrium Distribution of a Charge in a Homogeneous Chain with a Defect
}

\author{
Nadezhda Fialko ${ }^{1, \star}$, Maxim Pyatkov ${ }^{1}$, and Victor Lakhno ${ }^{1}$ \\ ${ }^{1}$ Institute of Mathematical Problems of Biology RAS - the Branch of Keldysh Institute of Applied Mathematics \\ of Russian Academy of Sciences
}

\begin{abstract}
Based on the semi-classical Holstein Hamiltonian we consider charge transfer along a DNA chain of sites at different thermostat temperatures. Recently, using the computer simulation, it has been shown that the charge distribution in homogeneous chains in thermodynamic equilibrium depends not only on the temperature, but also on the length of the chain. We have studied numerically the case of polyadenine fragments with a defect site in the middle of the chain. The results demonstrate qualitatively similar behavior of thermodynamic equilibrium quantities in the case of the homogeneous chain and of the chain with a defect. Insertion of a trap-site enhances the stability of polaron states.
\end{abstract}

\section{Introduction}

The problem of charge or energy transfer in quasi-one-dimensional biomolecules is very interesting for such fields as biophysics and nanobioelectronics. In the 1970s, A.S. Davydov suggested that vibrational intramolecular excitations can be transferred along $\alpha$-helix of protein molecules by solitons without loss of energy [1]. The polaron mechanism of charge transfer attracts considerable attention of researchers due to its stability. Presently it is believed that the charge carriers in biopolymers, such as DNA, are polarons or solitons (see e.g. [2-4] and refs. therein). This view has been formed mainly due to the fact that DNA is a quasi-one-dimensional system in which excess charges - electrons or holes - evolve into polaron states in the case of their strong interaction with oscillatory degrees of freedom of a molecule. Today, there is a huge amount of modeling results, which demonstrate solitory waves moving along an unperturbed chain. On the temperature dependent polaron propagation, numerous computational experiments and theoretical investigations [5-8] allow one to conclude that a polaron disappears at a temperature lower then its binding energy.

In the papers $[9,10]$ the values of the total energy, electronic part of the energy and delocalized parameter in the thermodynamic equilibrium state were calculated for 1D homogeneous chains, and some common features were found. It was shown that it is not the temperature, but the thermal energy of the lattice subsystem that is the critical parameter that determines the polaron disruption. In this work we consider a homogeneous polyadenine chain with one defect site at the center, which plays the role of a trap for the charge. We simulate a model defect site and consider a simple but important case of a DNA fragment with an A... AGA... A sequence, where G plays the role of the 8-oxoguanine which is one of the most common DNA lesions resulting from hole migration along the chain [11].

\footnotetext{
^e-mail: fialka@impb.ru
} 
The resultant damage to DNA bases may be a significant source of mutations that lead to cancer and other human pathologies.

\section{Charge transfer model}

The model is based on the Holstein Hamiltonian for a discrete chain of sites [12]. In the semiclassical approximation, choosing the wave function $\Psi$ in the form $\Psi=\sum_{n=1}^{N} b_{n}|n\rangle$, where $b_{n}$ is the amplitude of the probability of the charge (electron or hole) occurrence at the $n$-th site $(n=1, \ldots, N, N$ is the chain length), we write down the averaged Hamiltonian:

$$
\langle\Psi|\hat{H}| \Psi\rangle=\sum_{m, n} v_{n m} b_{m} b_{n}^{*}+\frac{1}{2} \sum_{n} M \dot{\tilde{u}}_{n}^{2}+\frac{1}{2} \sum_{n} K \tilde{u}_{n}^{2}+\sum_{n} \alpha^{\prime} \tilde{u}_{n} b_{n} b_{n}^{*} .
$$

Here $v_{m n}(m \neq n)$ are matrix elements of the transition between the $m$-th and the $n$-th sites (depending on overlapping integrals), $v_{n n}$ is the electron energy at the $n$-th site. We use the nearest neighbor approximation, i.e. $v_{m n}=0$ if $m \neq n \pm 1$; the intrasite fluctuations $\tilde{u}_{n}$ are assumed to be small and can be considered to be harmonical; suppose that the probability of charge's occurrence at the sites depends linearly on the sites displacements $\tilde{u}_{n}, \alpha^{\prime}$ is the constant of coupling between quantum and classical subsystems, $M$ is the effective mass of the site, $K$ is the elastic constant.

Motion equations for Hamiltonian (1) have the form

$$
\begin{aligned}
& i \hbar \dot{b}_{n}=v_{n, n-1} b_{n-1}+v_{n, n+1} b_{n+1}+v_{n, n} b_{n}+\alpha^{\prime} \tilde{u}_{n} b_{n}, \\
& M \ddot{\tilde{u}}_{n}=-K \tilde{u}_{n}-\alpha^{\prime}\left|b_{n}\right|^{2}-\gamma \dot{\tilde{u}}_{n}+\xi_{n}(t) .
\end{aligned}
$$

To model a thermostat, subsystem (3) involves the term with friction ( $\gamma$ is a friction coefficient) and the random force $\xi_{n}(t)$ such that $\left\langle\xi_{n}(t)\right\rangle=0,\left\langle\xi_{n}(t) \xi_{m}(t+s)\right\rangle=2 k_{B} T \gamma \delta_{m n} \delta(s)$ (T is the temperature [K]). This way of imitating the environmental temperature with the use of Langevin equations (3) is well known [7, 13].

We calculated a set of samples using 2o2s1g-method [14]. Each sample is a trajectory of system (2)-(3) with its own initial data and random values of $\left\{x i_{n}(t)\right\}$. Having obtained a number of samples we find averaged "over ensemble" functions of time, and for big time interval of integration we can estimate thermodynamic parameters. We are interested in what the delocalization parameter

$$
\langle R\rangle=\left\langle\frac{1}{\sum_{n}\left|b_{n}\right|^{4}}\right\rangle
$$

will be when the system reaches thermodynamical equilibrium. Obviously, if a charge is localized at one $k$-th site, i.e. charge probability $\left|b_{k}\right|^{2} \sim 1$, then $R \sim 1$. For homogeneous polyA chains, the parameter values of which correspond to a small-radius polaron, in thermodynamic equilibrium at low $T\langle R\rangle \sim 1$ and for high $T\langle R\rangle \sim N / 2$.

For the homogeneous chains the electron energy values are the same for all sites, and we can take $v_{n n}=0$. When the chain has one defect site in the center, this site has negative electron energy $v_{n n}<0$ and serves as a trap for the charge. We have computed chains A... A X A... A with a model defect site $\mathrm{X}$ in the center for chain lengths $N=19$ and $N=40$, and a more realistic case: the Guanine is incorporated in the center of a polyA fragment. The model parameters corresponding to nucleotide pairs are chosen as follows $[15,16]$. The effective mass of a site is $M=10^{-21} \mathrm{~g}$; the rigidity $K=0.062 \mathrm{eV} / \AA^{2}$ and coupling constant $\alpha^{\prime}=0.13 \mathrm{eV} / \AA$. The matrix elements of the transition between sites are: $v_{A A}=0.030 \mathrm{eV}$, we choose the same $v_{A X}=v_{A A}$; also we choose the electron energy on A $v_{A}=0$; model traps are $v_{X}=-0.066 \mathrm{eV}$ and $v_{X}=-0.132 \mathrm{eV}$. For A... A G A...A fragments $v_{A G}=0.049 \mathrm{eV}, v_{G A}=0.089 \mathrm{eV}$, and the electron energy $v_{G}=-0.45 \mathrm{eV}$. 


\section{Results}

A qualitative picture of the $\langle R(T)\rangle$ values for such chains in thermodynamic equilibrium is similar to the results for a homogeneous chain without a defect [10]. When the thermal energy of the classical chain $E_{\text {class }}=N k_{B} T$ is less than the critical value $E_{\text {crit }}$, the charge is in the polaron state, $\langle R(T)\rangle \sim 1$. When $E_{\text {class }}>E_{\text {crit }}$, the charge is delocalized, $\langle R(T)\rangle \sim N / 2$.

If we rescale abscissa, i.e., choose the thermal energy of the classical chain instead of the temperature $T$ as variable $x, x=E^{*} N T$ ( $E^{*}$ is a dimensionless coefficient related to $k_{B}$ ), the values of $\langle R(x)\rangle$ for different values of $N$ are found to be close to the same curve. Figure 1 a) shows the $\langle R(x)\rangle$ curves for homogeneous polyA and for chains with the defect site. Low parts of graphs of Figure 1 a) correspond to polaron state, $\langle R\rangle \sim 1$. The greater is the depth of the defect $v_{X}$, the greater is the value of $E_{\text {crit }}$; i.e. for fixed $N$ the stability of the polaron state increases with increasing electron energy on the defect site $\left|v_{X}\right|$.

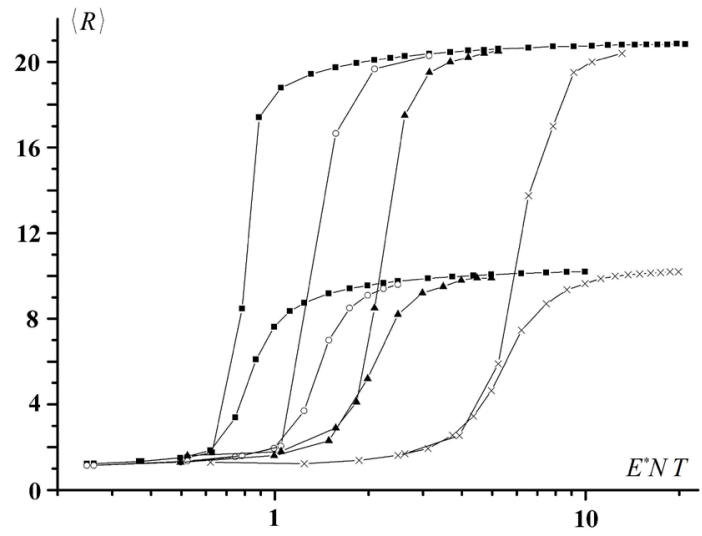

a)

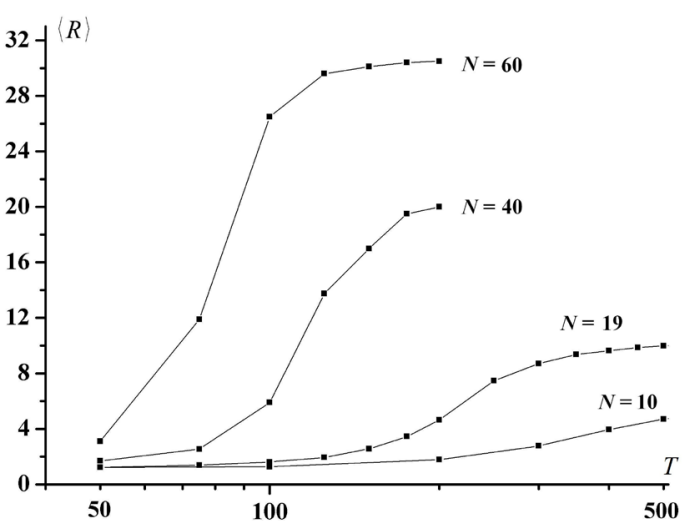

b)

Figure 1. Left: Dependencies of delocalization parameter on the energy of the classical chain. The graphs show two chain lengths $N=19$ and $N=40$ sites. Squares denote $\langle R\rangle$ values for homogeneous chains, circles show $\langle R\rangle$ for polyA with defect site $v_{X}=-0.066 \mathrm{eV}$, triangles - for polyA with defect site $v_{X}=-0.132 \mathrm{eV}$, and crossed lines correspond to A... A G A... A fragments, $v_{G}=-0.45 \mathrm{eV}$. Right: $\langle R(T)\rangle$ for the A... A G A... A fragments of different lengths $N$.

Figure $1 \mathrm{~b}$ ) shows the temperature dependencies $\langle R(T)\rangle$ for the A... A G A... A fragments of different lengths. It is shown that, e.g. for the same temperature $T=200$ : the polaron state exists in the "short" chain of 10 sites; the charge is in an intermediate state in the chain of 19 sites (the charge is localized about half the time in a sample); and the charge is delocalized in the "long" chains $N=40$ and $N=60$. For homogeneous polyA fragments, in TDE the polaron state exists at low temperatures, less than $5 \Theta(\Theta$ is Debye temperature, below which the semiclassical approximation is inapplicable). For the oligonucleotide AAAAGAAAAA $(N=10)$, the critical temperature $T_{\text {crit }}$ of polaron disruption is $T_{\text {crit }} \sim 400 \mathrm{~K}$, and for A... A G A...A fragment of 19 sites $T_{\text {crit }} \sim 300 \mathrm{~K}$ lies in the region of biological temperatures.

\section{Conclusions}

The qualitative picture for a homogeneous chain with a defect site is the same as for the chain without defect. The temperature stability of polarons in a chain with a defect site increases in comparison 
with the case of a homogeneous chain. The simulation results demonstrate that as the length of the chain increases, the type of charge distribution changes. Apparently, in short chains, the charge is in the polaron state, and its transfer occurs slowly by a hopping mechanism $[3,4,17]$. In long chains at the same temperature, the charge is in delocalized state, and the transfer process is more rapid.

The polaron localized on guanine can create 8-Hydroxyguanine, an abundant form of oxidative DNA damage, causes $\mathrm{G} \rightarrow \mathrm{T}$ and $\mathrm{C} \rightarrow \mathrm{A}$ substitutions [11]. The results obtained demonstrate that we can reduce the damaged guanine by changing the length of the chain or the sequence which leads to a decrease in the critical temperature of its delocalization. This can be interesting for making protectors from a DNA damage.

\section{Acknowledgments}

We are grateful to the HybriLIT group of JINR for computational resources.

The work is partially supported by Russian Foundation for Basic Research, grants no. 16-0700305, 17-07-00801, and Russian Science Foundation, grant 16-11-10163.

\section{References}

[1] A.S. Davydov, Journal of Theoretical Biology 66 (2), 379-387 (1977)

[2] E.M. Conwell, PNAS 102 (25), 8795-8799 (2005)

[3] G.B. Schuster (ed.), Long-Range Charge Transfer in DNA II, Topics in Current Chemistry 237 (Springer, 2004), $245 \mathrm{p}$.

[4] E.B. Starikov, S. Tanaka, and J.P. Lewis (eds.), Modern Methods for Theoretical Physical Chemistry of Biopolymers (Elsevier Scientific, Amsterdam, 2006) 461 pp.

[5] S. Komineas, G. Kalosakas, and A.R. Bishop, Phys. Rev. E 65 (6), 061905 (2002)

[6] L. Cruzeiro-Hansson and S. Takeno, Phy. Rev. E 56 (1), 894 (1997)

[7] P.S. Lomdahl and W.C. Kerr, Phys. Rev. Lett. 55 (11), 1235-1238 (1985)

[8] V.D. Lakhno, International Journal of Quantum Chemistry 108 (11), 1970-1981 (2008)

[9] V.D. Lakhno and N.S. Fialko, JETP 120, 125-131 (2015)

[10] N.S. Fialko, E.V. Sobolev, and V.D. Lakhno, JETP 124 (4), 635-642 (2017)

[11] K.C. Cheng, D.S. Cahill, H. Kasai, S. Nishimura, and L.A. Loeb, J. Biol. Chem. 267 (1), 166$172(1992)$

[12] T. Holstein, Annals of Physics 8, 325-342 (1959)

[13] E. Helfand, J. Chem. Phys 69, 1010-1018 (1978)

[14] H.S. Greenside and E. Helfand, Bell System Tech. J. 60, 1927-1940 (1981)

[15] A. A. Voityuk, J. Jortner, M. Bixon, and N. Rosch, J. Chem. Phys. 114 (13), 5614-5620 (2001)

[16] F.D. Lewis and Ya.Wu, J. Photochem. Photobiol. C 2 (1), 1-16 (2001)

[17] M. Bixon and J. Jortner, J. Phys. Chem. B. 104 (16), 3906 (2000) 